\title{
Design and performance of a programmable-temperature scanning tunneling microscope
}

M. S. Hoogeman, D. Glastra van Loon, R. W. M. Loos, H. G. Ficke, E. de Haas, J. J. van der Linden, H.

Zeijlemaker, L. Kuipers, M. F. Chang, M. A. J. Klik, and J. W. M. Frenken

Citation: Review of Scientific Instruments 69, 2072 (1998);

View online: https://doi.org/10.1063/1.1148901

View Table of Contents: http://aip.scitation.org/toc/rsi/69/5

Published by the American Institute of Physics

\section{Articles you may be interested in}

The ReactorSTM: Atomically resolved scanning tunneling microscopy under high-pressure, high-temperature catalytic reaction conditions

Review of Scientific Instruments 85, 083703 (2014); 10.1063/1.4891811

The ReactorAFM: Non-contact atomic force microscope operating under high-pressure and high-temperature catalytic conditions

Review of Scientific Instruments 86, 033706 (2015); 10.1063/1.4916194

Scanning probe microscopes go video rate and beyond

Review of Scientific Instruments 76, 053710 (2005); 10.1063/1.1915288

A cryogen-free low temperature scanning tunneling microscope capable of inelastic electron tunneling spectroscopy

Review of Scientific Instruments 87, 063701 (2016); 10.1063/1.4952577

Note: Development of a wideband amplifier for cryogenic scanning tunneling microscopy

Review of Scientific Instruments 88, 066109 (2017); 10.1063/1.4990041

A scanning tunneling microscope with a scanning range from hundreds of micrometers down to nanometer resolution

Review of Scientific Instruments 83, 103903 (2012); 10.1063/1.4744931

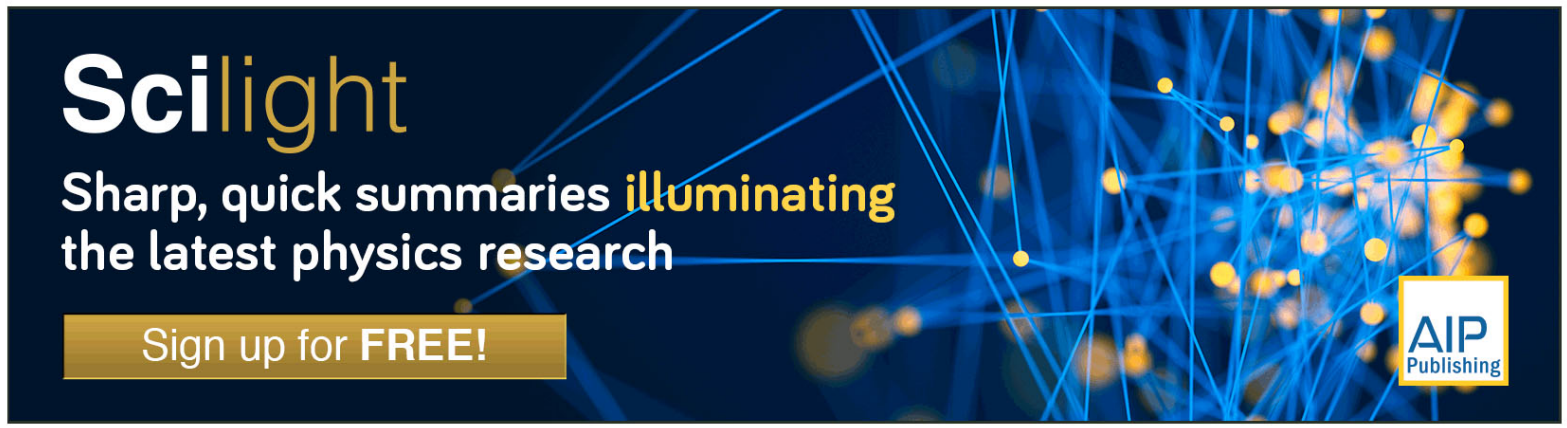




\title{
Design and performance of a programmable-temperature scanning tunneling microscope
}

\author{
M. S. Hoogeman, D. Glastra van Loon, R. W. M. Loos, H. G. Ficke, E. de Haas, \\ J. J. van der Linden, H. Zeijlemaker, L. Kuipers, ${ }^{a)}$ M. F. Chang, and M. A. J. Klik \\ FOM-Institute for Atomic and Molecular Physics, Kruislaan 407, 1098 SJ Amsterdam, The Netherlands \\ J. W. M. Frenken \\ Kamerlingh Onnes Laboratory, Leiden University, P. O. Box 9506, 2300 RA Leiden, The Netherlands and \\ FOM-Institute for Atomic and Molecular Physics, Kruislaan 407, 1098 SJ Amsterdam, The \\ Netherlands
}

(Received 29 October 1997; accepted for publication 17 February 1998)

\begin{abstract}
In this article we introduce a novel scanning tunneling microscope (STM), which operates in a sample temperature range from 60 to at least $850 \mathrm{~K}$. The most important new feature of this STM is that, while one selected part of the surface is kept within the microscope's field of view, the sample temperature can be varied over a wide range of several hundreds of degrees during actual imaging. The extremely low drift of the scanner and sample was achieved by the combination of a thermal-drift compensated piezoelectric scanner design with a newly developed sample stage. The design of the sample stage defines a fixed center from which thermal expansions, in all three directions, are forced outwards. The performance of the microscope is demonstrated for several surfaces including $\mathrm{Au}(110)$, on which we follow one particular surface region over a temperature range of more than 270 K. C) 1998 American Institute of Physics. [S0034-6748(98)02105-4]
\end{abstract}

\section{INTRODUCTION}

\section{A. Motivation}

Temperature is a key parameter in many surface properties. Examples are surface phase transitions, ${ }^{1-5}$ surface diffusion, ${ }^{6-11}$ and crystal growth phenomena. ${ }^{12,13}$ Nevertheless, one of the most powerful real-space surface imaging instruments, the scanning tunneling microscope, is very susceptible to temperature variations of the specimen. Even modest thermal drifting distorts the images and usually makes it impossible to follow one object on the surface over an appreciable range of temperature. Even more catastrophically, severe thermal drifting of the surface towards or away from the tip, ends the measurement when the change in surface to tip distance exceeds the limited span of the piezoelectric actuator.

In recent years scanning tunneling microscopes (STMs) have been developed which allow imaging at temperatures well above or below room temperature. In these microscopes nondistorted images can be obtained after a sufficiently long thermal equilibration time. Efforts have been made to minimize the equilibration time and the thermal drift either by limiting the heat capacity and the total heating power, ${ }^{14-16}$ or by a proper choice of materials and a uniform heating of the entire microscope. ${ }^{17}$ The company Omicron has developed a variable-temperature STM which operates down to $25 \mathrm{~K}$. For narrow beam-shaped $\mathrm{Si}$ samples a maximum temperature has been reached of $1500 \mathrm{~K} .{ }^{15}$ For different temperature regimes and sample materials different sample holders have to be

\footnotetext{
${ }^{a)}$ Present address: Dept. of Applied Physics, Twente University, P.O. Box 217, 7500 AE Enschede, The Netherlands.
}

used. The company JEOL has constructed a variabletemperature STM which works for temperatures below and above room temperature. ${ }^{16}$ The samples are heated either by direct current or by an optional indirect heating holder with which they reach a temperature of $1175 \mathrm{~K}$.

A true optimization has been performed by Kuipers et al. ${ }^{18}$ They modeled the thermal behavior of their microscope by means of a finite element analysis. By maximizing the temperature difference between the sample and the piezoelectric scanner the thermal equilibration time was minimized. The drift perpendicular to the sample surface was reduced to $0.71 \mu \mathrm{m}$ over a temperature interval of $159 \mathrm{~K}$. However, the drift in the lateral direction over the same temperature interval still exceeded the $\pm 1.7 \mu \mathrm{m}$ range of the piezoelectric actuators. The STM in Ref. 18 was not designed for measurements below room temperature.

Voigtländer et al. ${ }^{14}$ reported a STM with which the same spot on the surface is kept in view over a temperature ramp of $100 \mathrm{~K}$. They minimized the perpendicular and lateral drift by reducing the necessary heating power and the distances over which expansions occur. As in the Omicron STM, this could only be achieved for small semiconductor samples that were locally heated by direct current, and cannot be reproduced on a bigger metal sample with indirect heating. Lyding et al. ${ }^{17}$ have built a variable-temperature STM which is heated and cooled as a whole. A proper choice of materials reduced the drift in the lateral plane to only $10 \AA / K$. These results were obtained for a non-ultrahigh vacuum (UHV) version of the microscope. The design has been adapted for use in UHV and for high temperature measurements of semiconductor samples heated by direct current. ${ }^{19}$

In summary, many STM designs incorporate some form 
of optimization of the thermal behavior to shorten the thermal equilibrium time and to lower the thermal drifting. It has been demonstrated that during measurements the sample temperature can be changed, without exceeding the span of the piezoelectric actuator. However, it is still not possible with existing equipment to change the temperature of a nonsemiconductor sample over an appreciable range, in UHV, while keeping the same surface area within the microscope's view.

In this article we describe the design and operation of a UHV-compatible, programmable-temperature STM. We show that during a sample temperature change of several hundreds of degrees the same area can be kept in view on a metal sample, independent of the necessary heating power.

\section{B. Specifications}

Our main objective was to build a UHV-compatible STM in which the sample temperature can be swept over roughly $300^{\circ}$ during actual imaging, while one particular area on the surface is kept within the microscope's field of view. The temperature sweep-range of $300^{\circ}$ guarantees that we can follow, e.g., a complete surface phase transition or the evolution of surface morphologies.

The ranges of our piezoelectric actuator are $1.2 \mu \mathrm{m}$ and $3.5 \times 3.5 \mu \mathrm{m}^{2}$ in the directions perpendicular and parallel to the surface. Thus the displacement in the perpendicular direction should be less than $40 \AA / \mathrm{K}$ and in the lateral direction less than $165 \AA / K$. Furthermore, the drift during heating or cooling should be constant in speed and direction, to enable one to keep pace with the displacements by offsetting the piezoelectric actuator voltages.

Although our STM is equipped with high-speed data acquisition, ${ }^{18}$ allowing image rates of 10 images/s, the hopping rate of diffusing adatoms on most surfaces at and above room temperature is far beyond the imaging speed of the STM. For metal surfaces with a typical activation energy $E_{\text {act }}$ of $0.3 \mathrm{eV}$ and an attempt frequency $\nu_{0}$ of $10^{12} \mathrm{~Hz}$, the hopping rate $\nu=\nu_{0} e^{-E_{\text {act }} / k_{B} T}$ of adatoms is of the order of $10^{7} \mathrm{~Hz}$ at room temperature. The hopping rate is practically zero on the time scale of the experiment only below $100 \mathrm{~K}$ ( $70 \mathrm{~K}$ for $E_{\text {act }}=0.2 \mathrm{eV}$ ). Thus, for single-atom diffusion, the STM should operate at low sample temperatures, down to 50 $\mathrm{K}$. On many surfaces, the characteristic times at $1000 \mathrm{~K}$ of most surface phase transitions and diffusion phenomena are much shorter than the time resolution of our STM of 1 ms. ${ }^{9,18}$ Therefore, we have strived for a maximum temperature of $1000 \mathrm{~K}$ in the present design.

For growth and adatom diffusion studies we need to deposit the particles during the actual measurement. Therefore, the sample surface should be accessible to a molecular beam, also after the tip is brought within the $1.2 \mu \mathrm{m}$ range of the piezoelectric actuator. In addition, it should be possible to monitor the tip to sample distance during the coarse approach with an optical microscope.

The STM has to work both on semiconductor and metal samples. Our metal samples are heated indirectly, e.g., with a filament from the back. To allow for a high-quality mechanical polish within $0.05^{\circ}$ of a particular surface orientation the

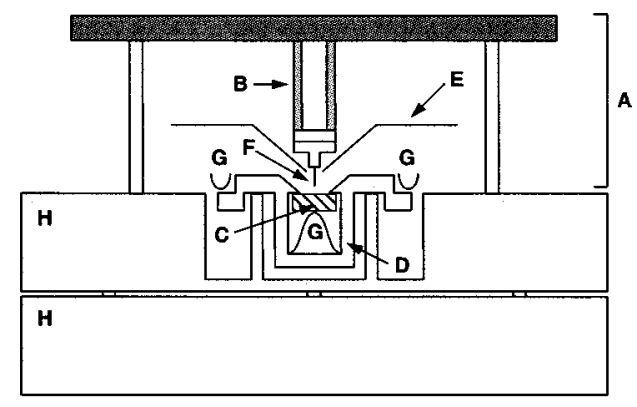

A scanner, B piezo element, C sample, D sample holder, $\mathbf{E}$ radiation shield, $\mathbf{F}$ tip, $\mathbf{G}$ leaf springs, H support block

FIG. 1. Schematic cross section of the programmable-temperature STM. The scanner $\mathbf{A}$ is cylindrically symmetric around the axis through the tip $\mathbf{F}$. It rests with three legs on the support block $\mathbf{H}$. A radiation shield $\mathbf{E}$ protects the piezo element $\mathbf{B}$ against thermal radiation from the sample $\mathbf{C}$. The sample holder $\mathbf{D}$ is clamped down against two supports by leaf springs $\mathbf{G}$. The sample is clamped up against two ledges of the sample holder.

dimensions of our metal samples cannot be smaller than 5 $\times 5 \times 1 \mathrm{~mm}^{3}$. Due to their size and type of heating the metal samples require significantly more heating power than beamshaped semiconductor samples with direct current heating. Nevertheless, we require the temperature range and sweep specifications to apply to metal samples.

To allow for multiple experiments, the sample holder should be an exchangeable unit. It has to be positioned in the microscope in a reproducible way, without changing the drift properties each time the sample holder is put into the microscope. The piezoelectric scanner should also be exchangeable to allow ex situ tip replacements. ${ }^{18}$ To measure on macroscopically different places on the sample, we require a lateral coarse positioning of the piezoelectric scanner. Two wobble sticks and a transfer rod are available to transfer the sample holder and the scanner between the UHV chamber and the loading chamber.

The remainder of this article is organized as follows. Section II explains how we reduced the thermal drifts of the sample and tip to a minimum. The experimental setup is described in Sec. III. The performance is demonstrated in Sec. IV. In Sec. V we give an outlook of further improvements of the present design.

\section{DESIGN}

\section{A. Basic configuration}

Figure 1 shows a schematic cross section of the microscope. The main body is a molybdenum block, which is the sample holder support. The molybdenum support block actually consists of two separate pieces which are thermally isolated from each other by three stainless steel capillaries. The sample holder is mounted in the upper piece and two piezo inertial drive motors are mounted in the bottom piece. On top is the scanner with the piezoelectric actuator and the tip. For the scanner we used the thermal-drift compensated scanner design of Kuipers et al. ${ }^{18}$

In the scanner a radiation shield protects the piezo element against thermal radiation from the sample. Apart from its three legs the scanner is cylindrically symmetric around 
the axis of the tip. A finite-element analysis of the scanner has been used to match the expansion of the legs with that of the assembly of piezo element, tip holder, and tip in order to minimize the vertical drift of the tip due to temperature changes of the sample. In addition, near matching has been obtained of the lateral expansion of the top plate of the scanner and the block supporting the scanner's legs (see Ref. 18).

The sample is clamped up in a molybdenum holder with its surface against two ledges. The holder itself is clamped down with two extending arms on two supports. The ledges, the arms, and the supports all lie in the same plane coinciding with the tip position. This configuration ensures that expansions of the sample and the holder do not affect the vertical position of the surface plane with respect to the tip.

The sample is radiatively heated from below by a filament. The filament is held inside the sample holder and shielded in all directions by the walls of the holder and the sample. In addition to radiative heating, the sample can be heated by electron bombardment from the filament to the back of the sample.

Due to the open design of the STM, the sample surface is accessible during the actual measurement from two sides, e.g., for a molecular beam and an optical microscope. The maximum angle of incidence is $30^{\circ}$ with the sample surface.

\section{B. Drift reduction in lateral plane}

The biggest challenge of the design is the thermal drift reduction between the sample and the tip in the lateral direction. As already mentioned in Sec. I B, the sample should not drift out of the range of the piezoelectric actuator over a sample temperature span of several hundreds of degrees. Furthermore, residual displacements during heating or cooling should be constant in speed and direction, so that we can easily adjust for the drift by offsetting the piezoelectric actuator voltages.

The cylindrical symmetry of the scanner, with respect to the tip and the near match between the lateral expansion of the top plate of the scanner and the support block, guarantees that the lateral drift of the tip is minimal. The most important new feature in our present design is the drift reduction of the sample holder. Figure 2(a) shows a schematic top view of the holder. Four arms extend from the holder. Two of these are rotated against vertical posts that form part of the Mo support block. The other two are shaped as knife edges and are pressed down against two flat supports by two leaf springs. When the holder is heated it expands outwards along the four extensions but the center stays at its original position. To make the coarse approach possible the tip is mounted $1 \mathrm{~mm}$ outside this stable center position (see Sec. III B).

The four points of contact, the two knife edges and the two arms, seem to overdefine the position of the sample holder. To avoid the overdefinition, we first let the arms of the sample holder touch the posts when the holder is pushed into the block. At this stage the sample holder still has the freedom to rotate around the axis through these first two contact points. The rotation freedom ensures that both knife edges will make contact with the flat supports when the holder is pushed further. In this way, a completely unstrained

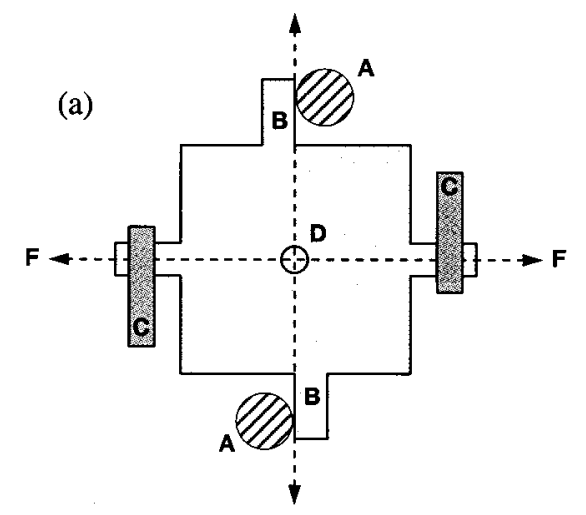

(b)

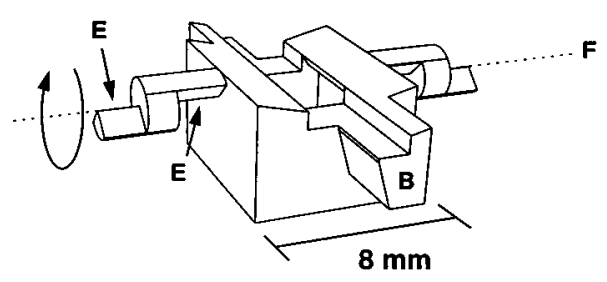

A post, B arm, C leaf spring, D stable center, E knife edge, $F$ rotation axis

FIG. 2. (a) Schematic top view of the sample holder body. The center circle D denotes the point from which all the lateral expansions are directed outwards. (b) Perspective drawing of the sample holder. The sample holder can rotate around the axis $\mathbf{F}$, defined by the knife edges $\mathbf{E}$.

four-point mechanical contact is established. Once in place, the sample holder can still rotate around the two knife edges, which is used for the coarse approach described in Sec. III B [see Fig. 2(b)]. To prevent random rotation of the sample holder due to the thermal expansion of the leaf springs, the springs press on two additional knife edges. These knife edges point upwards and lie on the same line as the rotation axis such that no torque can be exerted on the holder by the springs.

The holder is made of molybdenum. This material has a modest expansion coefficient of $4.8 \times 10^{-6} \mathrm{~K}^{-1}$ and a high melting point of $2890 \mathrm{~K}$ Furthermore, molybdenum does not tend to alloy with most of the other metals and semiconductors used in our experiments. The size of the sample holder is kept small $\left(8 \times 10 \times 10 \mathrm{~mm}^{3}\right)$, to minimize the length over which thermal expansions can occur. The sample itself is pushed up by a spring against two ledges of the sample holder (see Fig. 1).

The difference in expansion between the sample material and the molybdenum sample holder body results in a lateral displacement of the sample with respect to the holder during heating. We have tried to control this displacement by means of a radial pattern of fine ridges, which was spark eroded in the ledges of the holder against which the sample is pressed. The (extrapolated) center of this star pattern coincided with the tip position. Our hope was that the sample would expand radially along the ridges of the star pattern, keeping its center in place. Test measurements, however, revealed that the sample always expands away from a point somewhere along one of the two ledges where it happens to be attached most 


\section{(a)}

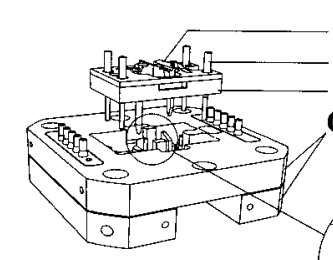

(b)

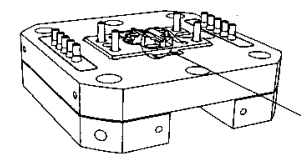

(c)
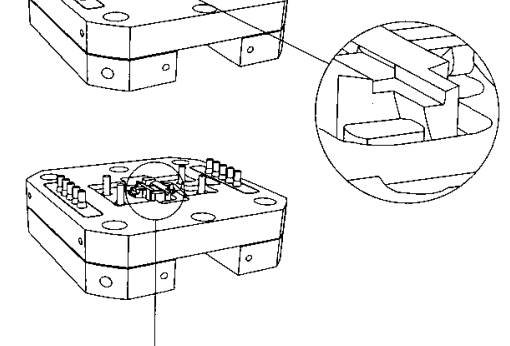

G D

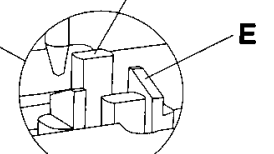

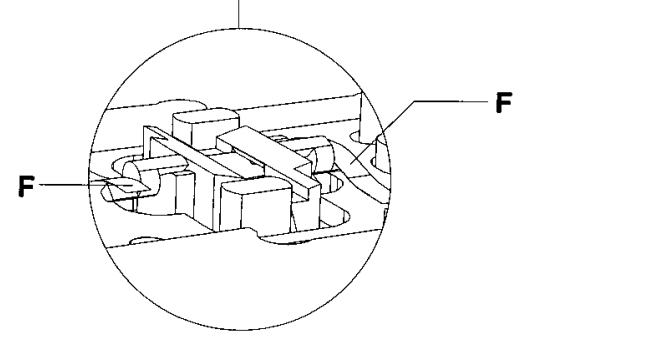

A sample holder body, B Burndy connector, C sample holder frame, D post, E support, F leaf spring, $\mathbf{G}$ support block

FIG. 3. Diagram of the assembly during insertion of the sample holder. In (a) the sample holder frame $\mathbf{C}$ and body $\mathbf{A}$ are still above the support block G. The sample holder body first touches, with its arms, the two posts $\mathbf{D}$ in the block (b). The leaf springs $\mathbf{F}$ push the body downward along the two posts, until the two knife edges touch the two supports E. In (c) the sample holder is fully inserted.

strongly to the holder. To ensure that this point is within 1 $\mathrm{mm}$ of the tip position we press the sample against two bumps on the sample holder ledges and an additional molybdenum "hook." The hook is $1 \mathrm{~mm}$ and the two bumps are each $2 \mathrm{~mm}$ away from the tip position.

The final sample holder consists of two parts: the sample holder body (as described above) and the sample holder frame (which is around the sample holder body) [see Fig. 3(a)]. The frame carries five gold-coated copper pins (Burndy) ${ }^{20}$ for the electrical connections to the thermocouple and the filament. The five corresponding sockets are mounted in the Mo support block. Also mounted on the frame are the two leaf springs, which press the sample holder body down.

\section{EXPERIMENTAL SETUP AND PROCEDURES}

\section{A. Sample holder exchange}

The UHV system is equipped with a wobble stick which allows in situ exchange of the sample holder (body together with frame) and the scanner. If the sample holder is being inserted into the block, the wobble stick lowers the frame, while at the same time the two leaf springs push the sample holder body down with its arms along the two posts [Fig. 3(b)]. After both knife edges first touch the supports, the frame is lowered another $2 \mathrm{~mm}$ to increase the spring load on the sample holder body and to decouple the body from the frame [Fig. 3(c)]. The surfaces of the two supports are tilted in opposite directions, such that the vertical spring force makes the sample holder body rotate clockwise, with its two arms against the vertical posts [Figs. 2(a) and 3(a)]. When the sample holder is not clamped in the block, the frame carries the sample holder body.

The support block rests with eight springs on a small carriage. The carriage is moved through the vacuum chamber over a monorail by use of a rotary feedthrough coupled to a chain. The carriage can be parked under several surface characterization and cleaning tools, e.g., a cylindrical mirror analyzer for Auger electron spectroscopy, a low-energy electron diffraction system, and a sputter ion gun. The entire chamber rests on an optical table, which is supported by air-cushioned legs.

\section{B. Coarse positioning}

The coarse approach is performed by rotating the sample holder body towards the tip until the sample surface is within the $1.2 \mu \mathrm{m}$ range of the piezoelectric actuator. The sample holder body rotates around the axis through the knife edges. The tip is positioned $1 \mathrm{~mm}$ away from this rotation axis. We can rotate the holder over $\pm 5^{\circ}$ which means that the tip has to be mounted in the tip holder with an accuracy of \pm 0.09 $\mathrm{mm}$. We rotate the sample holder body by pushing and pulling the bottom with an inertial piezo motor (see Sec. III C). The motor makes single steps of $\approx 0.5 \mu \mathrm{m}$ which brings the sample surface each step $0.05 \mu \mathrm{m}$ closer to the tip. The coarse approach is fully automated and is usually completed within $10 \mathrm{~min}$.

If the end point of the tip does not coincide precisely with the reference place defined by the upper surface of the support block, the sample holder will end up tilted with respect to the ideal horizontal orientation. At first sight this might seem to result in an uncompensated vertical distance in the sample holder and sample, of which the expansion or contraction would contribute directly to a vertical drift. However, since the support axis defined by the knife edges lies in the surface plane of the sample, the height of this plane is independent of temperature, even if this plane is tilted. The only uncompensated length in the case of a tip mounting error is the error in the tip length itself. Since the temperature variation of the tip is always more than one order of magnitude smaller than that of the sample, the resulting vertical shift is always below $0.5 \AA / K$.

A second inertial piezo motor is used for lateral coarse positioning of the scanner. This motor can shift the scanner over a range of $\pm 1 \mathrm{~mm}$ parallel to the rotation axis of the sample holder, thus keeping the distance between tip and rotation axis constant

\section{Inertial piezo motor}

The coarse positioning of the sample holder and the piezoelectric scanner is performed with an inertial piezo motor. The motor uses the stick-slip motion of a sliding mass over 
(a)

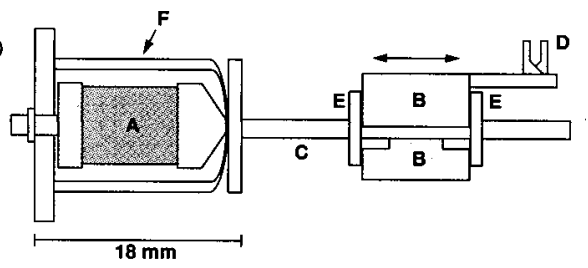

(b)

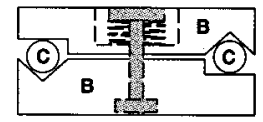

A piezoelectric actuator, $\mathbf{B}$ block, $\mathbf{C}$ guiding rods, D pins, E strips, $\mathbf{F}$ cylinder

FIG. 4. Schematic representation of the inertial piezo motor for rotating the sample holder body. The side strips $\mathbf{E}$ prevent the blocks from rotating. The two pins $\mathbf{D}$ push against (or pull at) the bottom side of the sample holder body, such that the translation of the blocks is converted into a rotation of the sample holder body. (a) Side view of the setup. (b) Cross section at the location of the slider.

two guiding rods. ${ }^{21}$ The motor consists of a piezoelectric actuator $^{22}$ and two blocks (the slider) clamped together around the two guiding rods (see Fig. 4). The piezoelectric actuator moves the guiding rods slowly forward and retracts them fast (or vice versa). The inertia of the two blocks makes them slide forward (or backward).

In order to avoid high friction and excessive wear even after extensive use under unlubricated, clean UHV conditions, one has to select a suitable material combination. For the blocks we use a soft material (stainless steel), coated with a $2 \mu \mathrm{m}$ gold film. A "hardcor" treatment ${ }^{23}$ was applied to the stainless steel guiding rods to harden their surface. The two blocks are held together with an adjustable push spring. A V-groove in each block defines the direction in which it can move. Four strips attached to the sides of the two blocks prevent them from rotating with respect to each other.

An important property of the inertial motor is its resonance frequency. The resonance frequency limits the fast part of the stick-slip cycle. To increase the resonance frequency the motor is made compact and light. The piezoelectric actuator is placed in a cylinder with a thin cap. To ensure that the actuator itself is not torn apart during fast contraction, it is pushed against the cap by a screw such that there is a permanent compression force of $200 \mathrm{~N}$ on the actuator. The guiding rods are directly fixed to the cap. The whole construction guarantees a resonance frequency above $1 \mathrm{kHz}$.

The motor is driven by single period wave forms with an amplitude of $625 \mathrm{~V}$ corresponding to a $3.1 \mu \mathrm{m}$ contraction or expansion of the piezo stack. The wave form consists of two mirrored half-parabolas, which are connected at their maxima. The parabola shape of the wave form ensures that the start and the end of the stick-slip cycle is smooth, while the return point is as sharp as possible (the bandwidth of the amplifier is $50 \mathrm{kHz}$ ). The piezo inertial motor makes steps of $\approx 0.5 \mu \mathrm{m}$ per wave form period.

We tested the inertial piezo motor in air and UHV. In air the motor can push and pull with a force of $2 \mathrm{~N}$. In UHV we could not measure the precise maximum push and pull force.
Over a period of 5 months we observed a modest decrease in average step size from 0.7 to $0.5 \mu \mathrm{m}$.

\section{Cooling}

We reach low sample temperatures by cooling the entire Mo support block in which the sample holder is clamped. Because the sample holder body is pushed by two leaf springs against the block, the sample holder reaches almost the same temperature as the block reaches (maximum difference of $6 \mathrm{~K}$ ).

The block is cooled by an Oxford Instruments flow cryostat. ${ }^{24}$ Our setup does not allow a permanent connection between the cryostat and the support block of the STM. Instead, we constructed a removable connection containing a large copper claw. The jaws of the claw have an opening that is $0.1 \mathrm{~mm}$ wider than the $80 \mathrm{~mm}$ width of the Mo support block. If the claw is cooled from room to liquid nitrogen temperature $(77 \mathrm{~K})$, the opening of the jaws shrinks 0.15 $\mathrm{mm}$, while the block shrinks $0.02 \mathrm{~mm}$ over the same temperature interval. The relatively large expansion coefficient of copper makes the claw clamp itself firmly around the molybdenum block, resulting in an excellent thermal connection. The claw is connected to the cryostat via a copper braid. We use an untwisted copper braid in order to minimize the transmission of vibrations originating from the cooling liquid. A thin plate of aluminum nitride $(0.75 \mathrm{~mm})$ between the braid and the cryostat electrically insulates the cryostat from the support block.

\section{PERFORMANCE}

\section{A. Displacement in lateral plane}

The thermal stability of the microscope in the lateral plane is illustrated in Fig. 5. The figure shows a sequence of differentiated STM images of a narrow island of monoatomic height on highly oriented pyrolytic graphite (HOPG). The time between subsequent images was $30 \mathrm{~s}$. During imaging we heated the sample from room temperature to $30 \mathrm{~K}$ above room temperature. We held the position of the scan fixed during the measurement. The average displacement over this temperature interval was $40 \AA / \mathrm{K}$. The displacement was mainly unidirectional and reversible in temperature: when the sample was brought back to the starting temperature the sample shifted back close to its original position.

The displacement is caused mainly by the difference in the expansion between the sample holder body and the sample itself. The magnitude of the shift per unit of temperature is the expansion-coefficient difference between sample and holder times the distance between the tip position and the point where the sample is fixed in the holder. A smaller contribution to the shift originates from the excentric position of the tip ( $1 \mathrm{~mm}$ away from the rotation axis). The expansion coefficients of graphite and molybdenum are 1.5 $\times 10^{-6}$ and $4.8 \times 10^{-6} \mathrm{~K}^{-1}$, respectively. From the measured shift and the expansion coefficients we calculate that the graphite sample is fixed at a location $1.9 \mathrm{~mm}$ away from the tip. The direction of the shift and the distance of $1.9 \mathrm{~mm}$ indicate that the fixing point is located on one of the ledges 

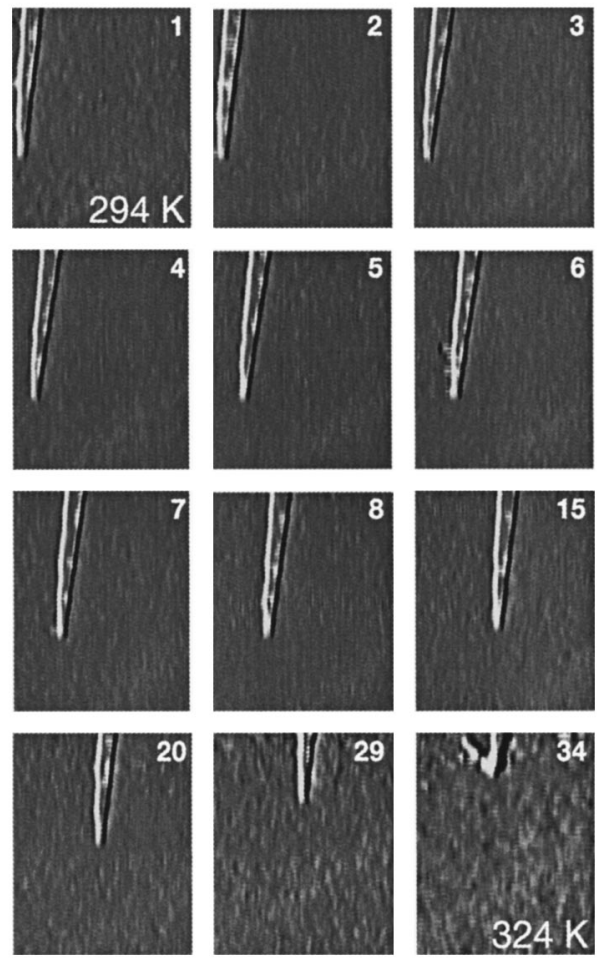

FIG. 5. Sequence of differentiated STM images of a section of a HOPG surface $(1840 \AA \times 2260 \AA$ ) during a temperature ramp from 294 to $324 \mathrm{~K}$. The scan position was not shifted. The images show an almost unidirectional drift of the surface of $40 \AA / K$. The numbers in the right-hand corner indicate the image number. The sudden change in the shape of the peninsula in the last image was due to a tip instability $\left(V_{t}=0.32 \mathrm{~V}, I_{t}=0.3 \mathrm{nA}\right)$.

in the sample holder body. Thus, the thermal displacement depends on the location where the sample is fixed and on the expansion coefficient of the sample.

If the sample temperature is changed the sample moves to a new position and it shifts back when the temperature is brought back to its original value. In view of its reversibility and unidirectional nature of the displacement, we refer to the displacements as thermal shifts rather than thermal drift. Long-term warming of the surroundings of the sample holder including the scanner during heating experiments hardly affects the drift behavior of the microscope.

The second example is a heating experiment on the $\mathrm{Ni}(100)$ surface. After a short equilibration at a sample temperature of $466 \mathrm{~K}$, the heating power was suddenly increased from 3.3 to $4.6 \mathrm{~W}$. The temperature and the thermal shifts in the $x$ and $y$ directions during the first $6 \mathrm{~min}$ following the step in heating power are shown in Fig. 6. After $84 \mathrm{~s}$ a maximum heating rate of $16 \mathrm{~K} / \mathrm{min}$ was reached. After $323 \mathrm{~s}$ the heating rate had reduced to $3.1 \mathrm{~K} / \mathrm{min}$. The shifting in $x$ and $y$ directions varied roughly proportionally with the heating rate. The near constant ratio between $d x / d t, d y / d t$ and $d T / d t$ and the absence of significant noise on the shifting illustrates the unidirectionality and reproducibility of the shifting behavior. If we sum the displacements in the $x$ and $y$ direction over all the images, we calculate a total displacement of $0.6 \mu \mathrm{m}$ and an average thermal shift of $163 \AA / \mathrm{K}$. Nickel has an expansion coefficient of $13.4 \times 10^{-6} \mathrm{~K}^{-1}$. The observed displacement corresponds to a distance of $1.9 \mathrm{~mm}$ between the tip and the point where the $\mathrm{Ni}$ sample is fixed

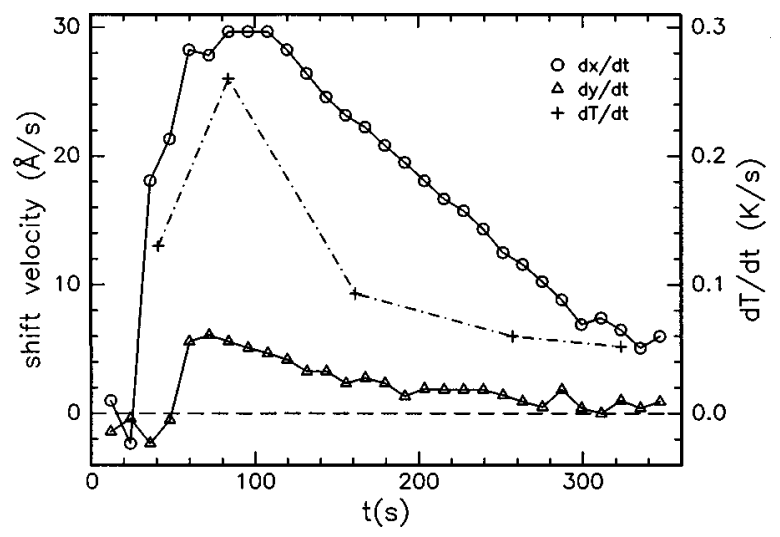

FIG. 6. Measured thermal shifts in the $x y$ plane between successive images vs time on $\mathrm{Ni}(100)$. Also the heating rate is indicated (right-hand axis). At $t=0 \mathrm{~s}$ the heating power was increased from 3.3 to $4.6 \mathrm{~W}$. The lateral displacements respond immediately to the temperature rise $\left(V_{t}=-0.31 \mathrm{~V}\right.$, $\left.I_{t}=-0.02 \mathrm{nA}\right)$.

within the Mo holder. Similarly, on $\mathrm{Au}(110)$, which has an expansion coefficient of $14.2 \times 10^{-6} \mathrm{~K}^{-1}$, we found a drift of $180 \AA / \mathrm{K}$, which corresponds to a distance between tip and fixing point of $1.9 \mathrm{~mm}$.

In a third example we demonstrate the reversible and unidirectional nature of the thermal shift, again on the $\mathrm{Ni}(100)$ surface. After a short equilibration time at a sample temperature of $440 \mathrm{~K}$ the heating power was suddenly increased from 3.5 to $3.8 \mathrm{~W}$ and after $300 \mathrm{~s}$ reduced back to $3.5 \mathrm{~W}$. Figure 7 shows the temperature and the thermal shifts in the $x$ and $y$ directions and the inset shows the $x$ and $y$ positions of the tip with respect to the sample during the time of this experiment. As previously, the shifting in $x$ and $y$ directions varies proportionally with the sign and the amplitude of the heating rate. The maximum displacement is 0.22 $\mu \mathrm{m}$ and the average thermal shift is $169 \AA / \mathrm{K}$, accordingly. Although we released the sample from the sample holder in between the two Ni experiments, we find for both experiments nearly the same magnitude and direction of the thermal shift. The inset of Fig. 7 shows that the sample shifted

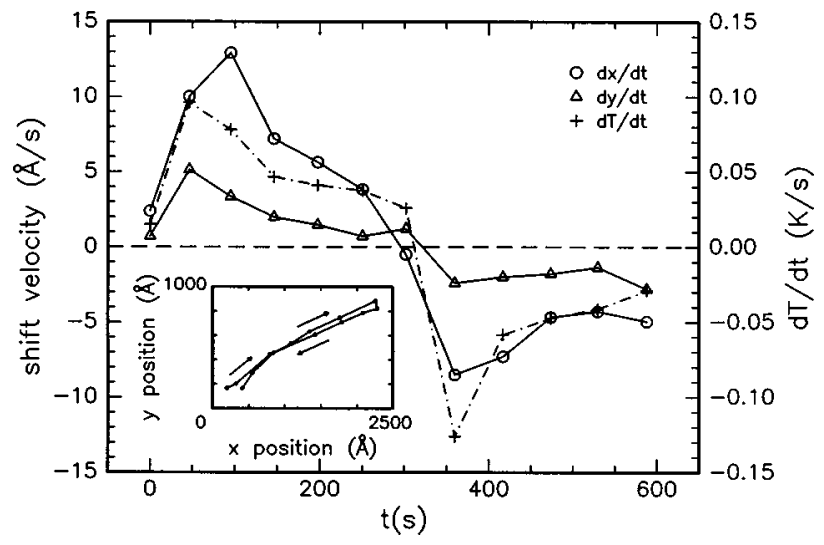

FIG. 7. Measured thermal shifts in the $x y$ plane between successive images together with the heating rate on $\mathrm{Ni}(100)$. The measured relative position of the sample is shown in the inset. At $t=0 \mathrm{~s}$ the heating power was increased from 3.5 to $3.8 \mathrm{~W}$ and at $t=300 \mathrm{~s}$ the heating power was reduced to $3.5 \mathrm{~W}$. The inset shows that after a maximum displacement of $2200 \AA$ at $453 \mathrm{~K}$ the sample shifts back to within $215 \AA$ from its starting position at $t=0 \mathrm{~s}\left(V_{t}\right.$ $\left.=-0.32 \mathrm{~V}, I_{t}=-0.02 \mathrm{nA}\right)$. 

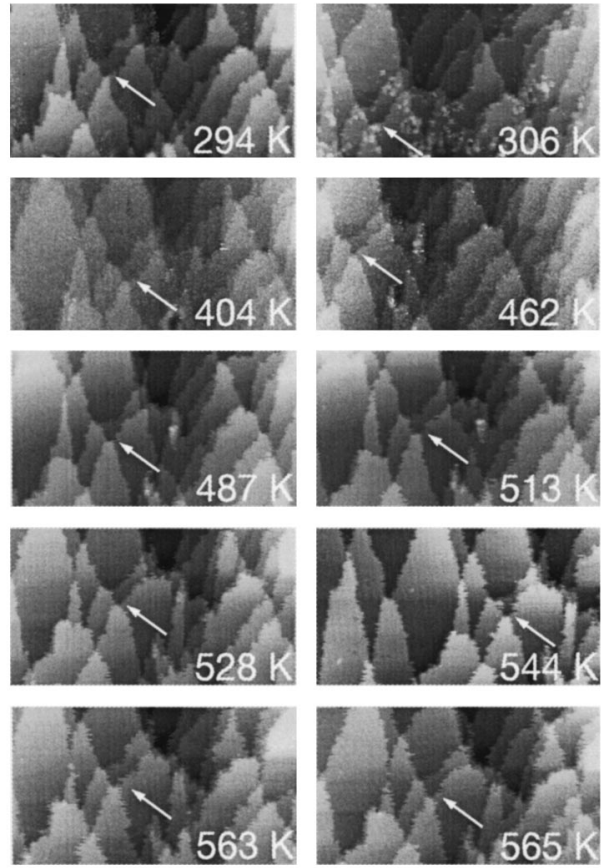

FIG. 8. Sequence of gray scale STM images of one particular surface region $(2490 \AA \times 1245 \AA)$ on $\mathrm{Au}(110)$ which is kept in view during a temperature ramp from 294 to $565 \mathrm{~K}$. The duration of the sequence was $120 \mathrm{~min}$ and the total drift of the surface was $\approx 4 \mu \mathrm{m}$. The contrast originates from monoatomic steps $(1.4 \AA)$. The vague lines in some of the images are a Moiré pattern between the scan lattice and the missing-row structure of the $\mathrm{Au}(110)$ terraces $\left(V_{t}=-0.69 \mathrm{~V}, I_{t}=-0.03 \mathrm{nA}\right)$. The arrow points to the location that we succeeded to keep in view.

back during the cooling, returning almost precisely along the same unidirectional trajectory as during the heating. The sample moved back to within $215 \AA$ of its original position after a maximum displacement of $2200 \AA$ at $453 \mathrm{~K}$.

Although the precise fixing point varies from sample to sample, the thermal shift is reproducible for each sample as long as it is not released from the sample holder. The predictable thermal shift of the sample makes it easy to anticipate during temperature ramps. Even in case the particular area of interest is lost, this place can always be found simply by going back to the temperature where the area was observed for the last time. Figure 8 shows a sequence of images of the $\mathrm{Au}(110)$ surface during a temperature ramp of $271 \mathrm{~K}$. We coarsely adjusted the center of each scan by means of offset voltages on the piezoelectric scanner. Over the whole temperature range one particular surface feature could be kept in view. The sum of the applied offset voltages corresponded to a total displacement of $\approx 4 \mu \mathrm{m}$ yielding an average drift of $150 \AA / K$. This drift is slightly lower than the value mentioned above. We attribute the difference to longterm warming of the surroundings and the scanner resulting in a small compensating drift. At elevated temperatures the monoatomic steps appear rugged. This is a consequence of the fast diffusion of the steps. ${ }^{89}$ Also, modest local morphology changes are visible during the temperature ramp.

\section{B. Drift in vertical direction}

In all experiments the vertical thermal drift in the microscope, measured in the range between 300 and $850 \mathrm{~K}$, was

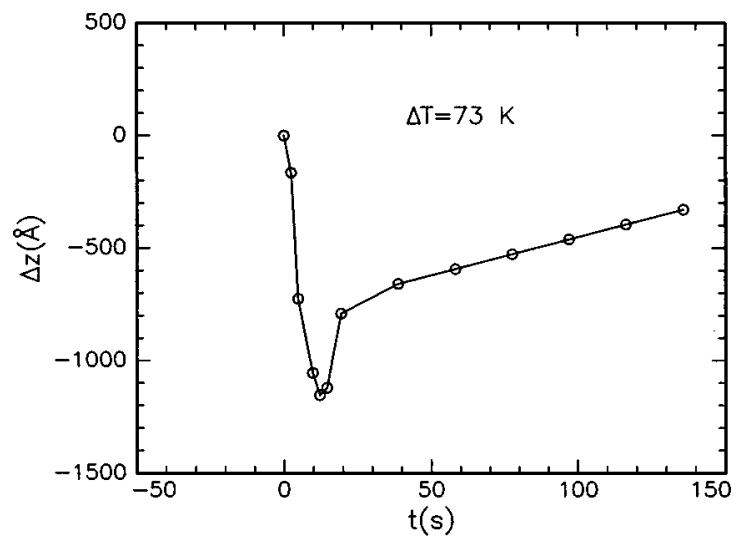

FIG. 9. Measured thermal drift in the $z$ direction between the tip and a $\mathrm{Ni}(100)$ surface due to an increase of the sample temperature. At $t=0 \mathrm{~s}$ we applied $1.8 \mathrm{~W}$ heating power, which corresponds to a temperature rise of the sample from room temperature to $370 \mathrm{~K}$. Initially, the surface and the tip drift away from each other with a speed $110 \AA / \mathrm{s}$ to a maximum shift of 1150 $\AA$. After $12 \mathrm{~s}$ the drift has reversed and after $135 \mathrm{~s}$ the net shift has decreased to $330 \AA$ at a sample temperature of $370 \mathrm{~K}\left(V_{t}=-0.46 \mathrm{~V}, I_{t}=\right.$ $-0.03 \mathrm{nA})$.

extremely low, namely $5 \AA / K$ or less. Figure 9 shows the vertical position of the tip measured on a $\mathrm{Ni}(100)$ surface. At $t=0 \mathrm{~s}$ we switched on the heating at a power of $1.8 \mathrm{~W}$. Initially, the surface and the tip drift away from each other with $110 \AA / \mathrm{s}$. After a short time of $12 \mathrm{~s}$ the drift direction reversed and after $135 \mathrm{~s}$ the net shift had reduced to $330 \AA$ at a sample temperature of $367 \mathrm{~K}$. If we switch off the heating we observe the opposite behavior.

Modest delays in heating or cooling are responsible for the transient behavior. First, the legs of the scanner heat up, causing the tip to drift away from the sample. Somewhat later, the piezoelectric actuator also warms up and compensates for the expansion of the scanner legs. The net drift is 5 $\AA / K$. The overshoot can be reduced by stepwise increasing the heating power and waiting in between the steps until the drift has reversed and reduced.

The extremely low vertical drift makes this STM ideal for investigating the evolution of local surface morphologies induced by temperature changes, as the temperature can be changed over a wide range without making it necessary to mechanically adjust the vertical distance. On $\mathrm{Ni}(100)$, for example, we have been able to follow the entire annealing process of a sputtered surface, from room temperature all the way up to $730 \mathrm{~K}$, without making any mechanical adjustments of tip or sample holder. ${ }^{25}$

Although we use the same piezoelectric scanner design as Kuipers et al., we find a significantly lower vertical drift than was reported in Ref. 18. Kuipers et al. already suggested that most of their vertical drift resulted from the lateral drifting of the sample holder with respect to its tilted support. The tilt was necessary in that design for the coarse approach, which consisted of pushing the sample holder up and down a ramp of $5^{\circ}$ with the horizontal plane. The new design of our sample holder and coarse approach mechanism has completely eliminated this problem, which has resulted in much more idealistic behavior of the microscope.

Until now the STM has been tested up to a sample temperature of $850 \mathrm{~K}$. For this temperature the required heating 


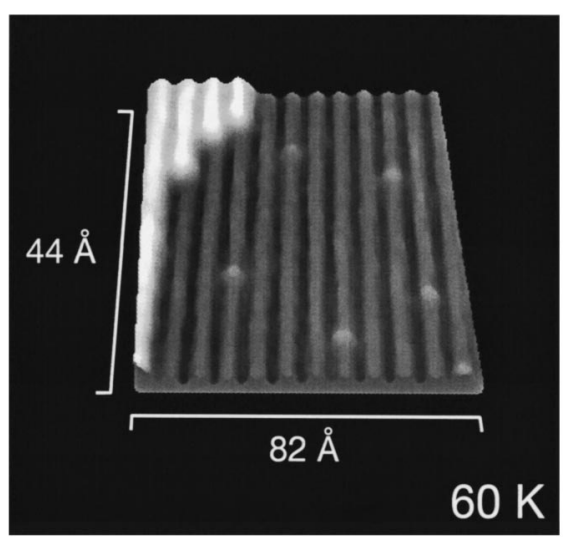

FIG. 10. Perspective representation of a STM image of a Au(110) surface, measured at $60 \mathrm{~K}$. The dark lines are the missing rows of the $(1 \times 2)$ reconstruction. The measured corrugation of the missing row structure is $0.7 \AA$. At the left we see three kinks of a higher terrace. The small dots on the lower terrace are adsorbates observed after in situ evaporation. The image was obtained in $5.4 \mathrm{~s}$. We measured a vibration amplitude introduced by the cooling of $<0.15 \AA\left(82 \AA \times 44 \AA, V_{t}=-0.69 \mathrm{~V}, I_{t}=-0.02 \mathrm{nA}\right)$.

power is $24 \mathrm{~W}$. Despite this high power the thermal drift behavior of the STM, both in the lateral and in the vertical plane, is not affected.

\section{Cooling and low temperature performance}

Although the present setup is not optimized for cooling, we reach a sample temperature of $60 \mathrm{~K}$ with liquid helium. In the present configuration the time needed to reach this temperature is quite long. The time to cool down from room temperature to $150 \mathrm{~K}$ is already $30 \mathrm{~min}$. The reason is our inefficient method of cooling. We cool the sample indirectly by cooling the entire upper piece of the Mo support block. The large heat capacity of the block causes the slow cooling rate. The high thermal radiation input of $3 \mathrm{~W}$ from the surroundings at room temperature limits the final temperature of the block to $55 \mathrm{~K}$. The sample holder is pushed against the block with leaf springs. The thermal contact is good enough to keep the temperature difference between sample holder and block within $6 \mathrm{~K}$. In a future design the sample holder will be mounted in a subsection of the Mo support block to which the braid is connected directly. This will reduce both the heat capacity and the thermal radiation input (see Sec. V).

The mechanical vibrations in the STM during cooling with liquid helium have an amplitude below $0.15 \AA$. Figure 10 shows a perspective representation of a STM image of $\mathrm{Au}(110)$ measured at $60 \mathrm{~K}$. The measured corrugation of the missing-row structure is $0.7 \AA$. The monoatomic step has a height of $1.4 \AA$. With liquid nitrogen we reach an end temperature of $130 \mathrm{~K}$. The vibration level is somewhat larger $(<0.4 \AA)$, but the missing-row structure and monoatomic steps on $\mathrm{Au}(110)$ can still be resolved with the STM.

\section{DISCUSSION AND OUTLOOK}

We have constructed a new programmable-temperature STM. The STM has been tested for temperatures between 60 and $850 \mathrm{~K}$. The novel STM has demonstrated its capability

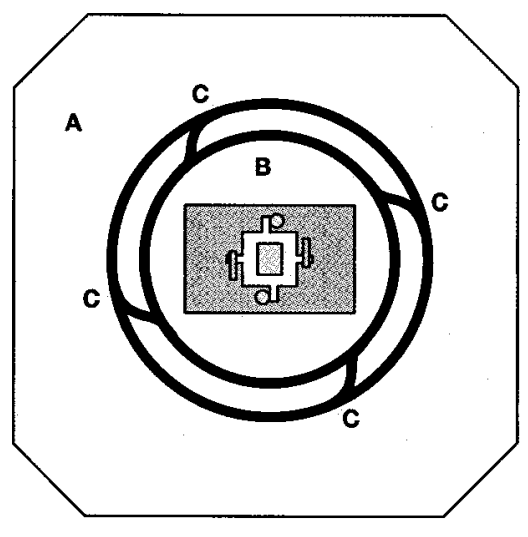

A outer section, $\mathbf{B}$ inner section, $\mathbf{C}$ bridges

FIG. 11. Schematic top view of the new support block design. The block is split in an outer section and an inner section. Four bridges connect the two sections. The sample holder sits in the inner section. If the inner section expands or shrinks with respect to the outer section, the center does not shift, but the inner section rotates around its center. The scanner is standing on the outer section.

of keeping the same area in view over a wide temperature range of at least $270 \mathrm{~K}$. Over a much wider interval the temperature can be changed during the measurement without the necessity for coarse vertical adjustments. The STM design is not optimized for cooling, but nevertheless we reach a sample temperature of $60 \mathrm{~K}$, using liquid helium.

The main future improvement of the STM design concerns the cooling system. The sample holder is cooled via the Mo support block, which has a large heat capacity and a high thermal radiation input. To minimize the temperature difference between the support block and the sample holder, a good thermal contact is required between both objects. A strong thermal coupling, however, is a disadvantage during operation at elevated sample temperatures. The heat of the sample holder leaks away to the block, thereby increasing the required heating power, and heating up the Mo support block. To improve the sample cooling we are adapting the design in collaboration with Oxford Instruments. ${ }^{26}$

The support block will be divided into an inner and an outer section. Figure 11 shows a schematic top view of the new block. The sample holder will be clamped in the inner section of the block and the scanner stands on the outer section. The inner section is separated from the outer section by four thin bridges $\left(5.0 \times 2.0 \times 0.5 \mathrm{~mm}^{3}\right)$. The cryostat will be directly connected to the inner section via a copper braid. To cool the sample in the new design, only the inner section has to be cooled instead of the whole block. The heat capacity of the inner section is approximately five times lower than that of the whole Mo block. The radiation input will be reduced from 3.2 to $0.6 \mathrm{~W}$. At a sample temperature of $50 \mathrm{~K}$, the heat conduction through the bridges will amount to $1.6 \mathrm{~W}$. In the present setup the heat input through conduction is negligible. Thus, the total heat input at a sample temperature of $50 \mathrm{~K}$ will be reduced from 3.2 to $2.2 \mathrm{~W}$. All the numbers reported were calculated assuming Invar as block material. Invar is an iron-nickel alloy and is known for its extremely low expansion coefficient and low thermal conductance. ${ }^{27}$ To ensure that the outer section stays within the temperature window in 
which the expansion coefficient is extremely low, we anchor the outer section via braids to the room temperature surroundings.

When the sample is heated above room temperature, the inner section will be heated up by the sample holder. In that situation the thin bridges again thermally isolate the inner section from the outer one. Therefore, the outer section together with the scanner will remain closer to room temperature during both heating and cooling.

If the sample is cooled or heated the inner section will contract or expand. Assuming that the spring constants of the bridges are equal and that the temperature profile is symmetric around the central axis, the inner section will not shift, but rotate around its center. We expect a rotation of $0.1 \mathrm{mrad}$ for a temperature difference of $200 \mathrm{~K}$ between the inner and outer section. The middle line of the bridges is aligned with the upper plane of the inner and outer section to prevent unwanted vertical drift of the inner section due to the strong temperature gradients over the bridges.

\section{ACKNOWLEDGMENTS}

The authors gratefully thank B. Ambrose and T. Palmer of Oxford Instruments ${ }^{26}$ (former WA Technology) for their collaboration during the design and testing of the programmable-temperature STM. ${ }^{28}$ They also acknowledge W. J. Barsingerhorn, J. ter Beek, R. Boddenberg, W. H. Brouwer, R. van Gastel, J. ter Horst, P. T. Keâ, E. de Kuyper, H. Neerings, M. Rost, and H. Voort for their assistance during the building of the microscope. R.J.I.M. Koper is also acknowledged for the preparation of the samples. This work is part of the research program of the Foundation for Fundamental Research on Matter (FOM) and was made possible by financial support from the Netherlands Organization for the Advancement of Research (NWO).

${ }^{1}$ J. Lapujoulade, Surf. Sci. Rep. 20, 191 (1994), and references therein.

${ }^{2}$ J. G. Dash, Contemp. Phys. 30, 89 (1989).

${ }^{3}$ J. W. M. Frenken, R. J. Hamers, and J. E. Demuth, J. Vac. Sci. Technol. A 8, 293 (1990).

${ }^{4}$ M. S. Hoogeman, D. C. Schlößer, J. B. Sanders, L. Kuipers, M. F. Chang,
M. A. J. Klik, D. Glastra van Loon, R. W. M. Loos, J. J. van der Linden, and J. W. M. Frenken, in Surface Diffusion: Atomistic and Collective Processes, NATO ASI Series B, Physics (Plenum, New York, 1997).

${ }_{5}^{5}$ J. W. M. Frenken, P. M. J. Marée, and J. F. van der Veen, Phys. Rev. B 34, 7506 (1986)

${ }^{6}$ G. L. Kellog, Surf. Sci. Rep. 21, 1 (1994), and references therein.

${ }^{7}$ R. Gomer, Rep. Prog. Phys. 53, 917 (1990).

${ }^{8}$ L. Kuipers, M. S. Hoogeman, and J. W. M. Frenken, Phys. Rev. Lett. 71, 3517 (1993).

${ }^{9}$ L. Kuipers, M. S. Hoogeman, J. W. M. Frenken, and H. van Beijeren, Phys. Rev. B 52, 11387 (1995).

${ }^{10}$ M. Giesen-Seibert, R. Jentjens, M. Poensgen, and H. Ibach, Phys. Rev. Lett. 71, 3521 (1993); 73, 911(E) (1994).

${ }^{11}$ T. R. Linderoth, S. Horch, E. Lægsgaard, I. Stensgaard, and F. Besenbacher, Phys. Rev. Lett. 78, 4978 (1997).

${ }^{12}$ Kinetics of Ordering and Growth at Surfaces, NATO ASI, edited by M. G. Lagally (Plenum, New York, 1990), Vol. 239.

${ }^{13}$ M. Zinke-Allmang, L. C. Feldman, and M. H. Grabow, Surf. Sci. Rep. 16, 377 (1992), and references therein.

${ }^{14}$ B. Voigtländer, A. Zinner, and T. Weber, Rev. Sci. Instrum. 67, 2568 (1996).

${ }^{15}$ General product information from Omicron Vakuumphysik GMBH, Idsteiner Straße 78, D-65232 Taunusstein, Germany.

${ }^{16}$ General product information from Jeol Ltd., 1-2 Musashino 3-chome, Akishima, Tokyo 196, Japan.

${ }^{17}$ J. W. Lyding, S. Skala, J. S. Hubacek, R. Brockenbrough, and G. Gammie, Rev. Sci. Instrum. 59, 1897 (1988).

${ }^{18}$ L. Kuipers, R. W. M. Loos, H. Neerings, J. ter Horst, G. J. Ruwiel, A. P. de Jongh, and J. W. M. Frenken, Rev. Sci. Instrum. 66, 4557 (1995).

${ }^{19}$ J.W. Lyding (private communication).

${ }^{20}$ Framatome Connectors Nederland bv, Essebaan 7, 2908 LJ Cappelle a/d IJssel, The Netherlands.

${ }^{21}$ Ch. Renner, Ph. Niedermann, A. D. Kent, and $\emptyset$. Fischer, Rev. Sci. Instrum. 61, 965 (1990).

${ }^{22}$ We used a PIC 151, UHV compatable, piezostack of Physik Instrumente (PI) GmbH \& Co., Polytec-Platz 5-7, 76337 Waldbronn, Germany.

${ }^{23}$ The "hardcor" treatment was carried out by Hardiff bv, P.O. Box 65, $7360 \mathrm{AB}$, The Netherlands.

${ }^{24}$ Oxford Instruments, Old Station Way, Eynsham, Witney Oxon OX8 1TL, United Kingdom.

${ }^{25}$ M. S. Hoogeman, M. A. J. Klik, R. van Gastel, and J. W. M. Frenken (unpublished).

${ }^{26}$ Oxford Instruments, SPM-group, Chesterton Mills, French's Road, Cambridge CB4 3NP, United Kingdom.

${ }^{27}$ General product information from Goodfellow Cambride Ltd., Cambridge, CB4 4DJ, United Kingdom.

${ }^{28} \mathrm{An}$ improved version of the programmable temperature STM described in this article is produced by Oxford Instruments, in Ref. 26. 